\title{
Antibacterial Activity of Sulfamethoxazole Transformation Products (TPs): General Relevance for Sulfonamide TPs Modified at the para Position
}

\author{
Marius Majewsky, ${ }^{* \dagger}$ Danny Wagner, ${ }^{\ddagger}$ Markus Delay, ${ }^{\dagger}$ Stefan Bräse, ${ }^{\ddagger} \S$ Viviane Yargeau, \\ and Harald Horn ${ }^{\dagger}$ \\ ${ }^{\dagger}$ Karlsruhe Institute of Technology (KIT), Engler-Bunte-Institut, Chair of Water Chemistry and Water Technology, \\ Engler-Bunte-Ring 1, 76131 Karlsruhe, Germany \\ ${ }^{\ddagger}$ Karlsruhe Institute of Technology (KIT), Institute of Organic Chemistry, Fritz-Haber-Weg 6, 76131 Karlsruhe, Germany \\ ${ }^{\S}$ Karlsruhe Institute of Technology (KIT), Institute of Toxicology and Genetics (ITG), Hermann-von-Helmholtz-Platz 1, 76344 \\ Eggenstein-Leopoldshafen, Germany \\ "Department of Chemical Engineering, McGill University, 3610 University Street, Montréal, Quebec J3N 1V3, Canada
}

Supporting Information

ABSTRACT: Sulfonamide antibiotics undergo transformation in the aquatic environment through biodegradation, photolysis, or hydrolysis. In this study, the residual antibacterial activity of 11 transformation products (TPs) of sulfamethoxazole (SMX) was investigated with regard to their in vitro growth and luminescence inhibition on Vibrio fischeri (30 min and $24 \mathrm{~h}$ exposure). Two transformation products, 4-hydroxy-SMX and $\mathrm{N}^{4}$-hydroxy-acetyl-SMX, were synthesized in-house and confirmed by nuclear magnetic resonance and high-resolution mass spectrometry. Results of individual compound experiments showed that TPs modified at the para amino group still exhibit clear antibacterial effects, whereas TPs resulting from breakdown of the SMX structure lost this mechanism of action. 4- $\mathrm{NO}_{2}$ - and 4-OH-SMX were found to inhibit growth to a clearly greater extent than the parent compound, SMX. In contrast, the $N^{4}$-acetyl- and $N^{4}$-hydroxy-acetyl-derivatives retain less than 10

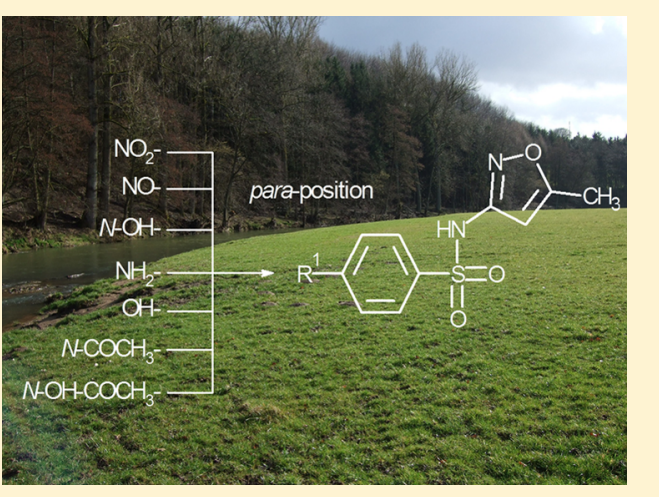
and $5 \%$ of the effect of SMX on growth and luminescence inhibition, respectively. The effect of a mixture of para-modified TPs was observed to be additive. Considering the homologous series of sulfa drugs widely prescribed and their common mechanism of action, the potential environmental impact must consider the total amount of sulfonamide antibiotics and their derivative TPs, which might end up in a water body. Extrapolating the results obtained here for the para TPs of SMX to other sulfa drugs and determining the persistence and occurrence of these compounds in the aquatic environment is required for improved risk assessment.

\section{INTRODUCTION}

Residues of pharmaceuticals and personal care products (PCPPs) are ubiquitously detected in urban wastewater and surface water alike. ${ }^{1}$ It recently has been demonstrated that PCPPs can adversely act on freshwater biofilms by respiration suppression, growth inhibition, and community alteration with momentarily unknown consequences for higher trophic levels and stream ecosystem functioning. ${ }^{2}$ In the context of the ongoing discussion about the risk assessment of the manifold transformation products (TPs) being formed, the question was raised as to whether TPs still exhibit the targeted mechanisms of action of the parent compound. ${ }^{3-5}$ In view of the frequent occurrence of sulfonamide antibiotics and due to their antimicrobial nature, the present study aims to address the (residual) ecotoxicological effects of sulfamethoxazole TPs. Sulfamethoxazole (SMX) is the most prominent short-acting representative of sulfonamide antibiotics used in high amounts in human and veterinary applications to treat and prevent bacterial infections of both Gram-positive and -negative species. It can be regarded as ubiquitous in urban wastewaters and is frequently detected in surface water. ${ }^{6,7}$

The bacteriostatic effect of sulfonamides on cell reproduction originates from the sulfanilamide toxicophore (sulfonamide group bound to aniline in the para postion, Figure 1) and is based on competitive enzyme inhibition and metabolic interference due to its similarity in molecular structure to $p$ aminobenzoic acid $(p \mathrm{ABA})$, an essential carboxylic acid involved in the natural intracellular folic acid synthesis of bacteria.

A number of recent studies reported different chemical or biological SMX TPs formed in aquatic processes on the lab

Received: July 7, 2014

Published: August 28, 2014 


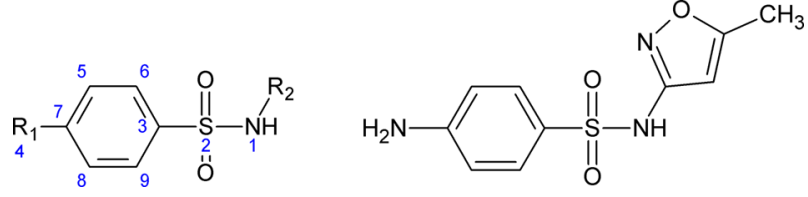

Figure 1. Generic structure, toxicophore group (with $\mathrm{R}^{1}=\mathrm{NH}_{2}$ ), and convention used in atom numbering of sulfonamide antibiotics (left) and structure of sulfamethoxazole (right).

scale, such as biodegradation, photolysis, or hydrolysis. ${ }^{8-15}$ However, while the ecotoxicity and identification of SMX TPs formed during wastewater ozonation has received increased attention, ${ }^{11,16}$ only limited information is available regarding the risk assessment of TPs from conventional natural transformation reactions. ${ }^{17}$ Additionally, sulfonamide TPs are rarely included in routine monitoring campaigns, which is why only scarce data are available about their occurrence. Initial occurrences of the nitro derivative 4-nitro-SMX and desaminoSMX were recently reported for spring samples in the nanograms per liter range. ${ }^{12}$ The $N^{4}$-acetylated form of SMX was detected up to $2.2 \mu \mathrm{g} \mathrm{L}^{-1}$ in a WWTP effluent and up to $235 \mathrm{ng} \mathrm{L}^{-1}$ in surface water downstream of wastewater discharge. ${ }^{18}$ Considering that numerous laboratory studies reported the formation of various TPs, it can be expected that other SMX TPs occur in aquatic environments. However, no information is currently available regarding a correlation between the residual antibiotic activity of TPs and their molecular structure.

Some of the TPs reported for SMX still contain a toxicophore-like moiety, and some derivatives incorporate almost the complete SMX parent compound structure. As a consequence, it is relevant and essential to determine the residual antibacterial activity of the manifold SMX TPs.

Previous studies on micropollutants revealed that, in most cases, the TPs are less toxic than the parent compound. ${ }^{19}$ However, some TPs exhibited similar or higher ecotoxicological effects than the parent compound and hence experimental testing remains indispensable. Moreover, with regard to the current concerns about antibiotic resistance in microbial biofilms ${ }^{20}$ the need to investigate the possible residual antibacterial potential of sulfonamide antibiotic TPs is apparent.

Addressing this need for a better assessment of the potential for sulfa drug TPs, the present study screened 11 TPs of SMX (Table 1) formed during human metabolism, microbial biodegradation, photolysis, and hydrolysis for their toxicity. TPs formed by the decomposition of SMX are referred to here as breakdown products, whereas TPs formed by transformations limited to the para position are denoted as derivatives. Formation processes reported in the literature and physicochemical properties for the selected SMX TPs are given in Table S1 (Supporting Information). Two metabolites, $N^{4}$-hydroxy-acetyl-SMX and 4-hydroxy-SMX, were synthesized in-house, as they were not commercially available. These were included in the study since they have been suggested to occur from biotransformation by activated sludge bacteria. ${ }^{10,21}$ Bacterial luminescence inhibition (LI) bioassays were selected to investigate the biological activity of the TPs. LI is commonly known to be a very sensitive and rather nonspecific toxicity end point. The bioassays were also carried out in growth medium, which ensures bacterial growth over a period of $24 \mathrm{~h}$. This change in methodology allows the bacteriostatic mechanism of action of sulfonamides to be evaluated by growth inhibition (GI) determination, providing a second toxicity end point.

The three research hypotheses considered in this study were that (i) SMX TPs retaining the sulfonamide toxicophore exhibit residual antibacterial properties and these can be related to their physicochemical properties, (ii) TPs not exhibiting the toxicophore are ecotoxicologically relevant via another mechanism of action, and (iii) these trends can be generalized to other homologous sulfonamide antibiotics to anticipate ecotoxicologically relevant TPs.

\section{EXPERIMENTAL PROCEDURES}

2.1. Chemicals. 3-Amino-5-methylisoxazole (CAS 1072-67-9), sulfanilic acid (CAS 121-57-3), sulfanilamide (CAS 63-74-1), benzenesulfonamide (CAS 98-10-2), aniline (CAS 62-53-3), and sulfamethoxazole (723-46-6) were purchased from Sigma-Aldrich (Oakville, ON, Canada). Acetyl-sulfamethoxazole (CAS 21312-10-7), NO-sulfamethoxazole (CAS 131549-85-4), N-OH-sulfamethoxazole (CAS 114438-33-4), and $\mathrm{NO}_{2}$-sulfamethoxazole (CAS 29699-89-6)

Table 1. Structures and Abbreviations of Sulfamethoxazole Transformation Products ${ }^{a}$

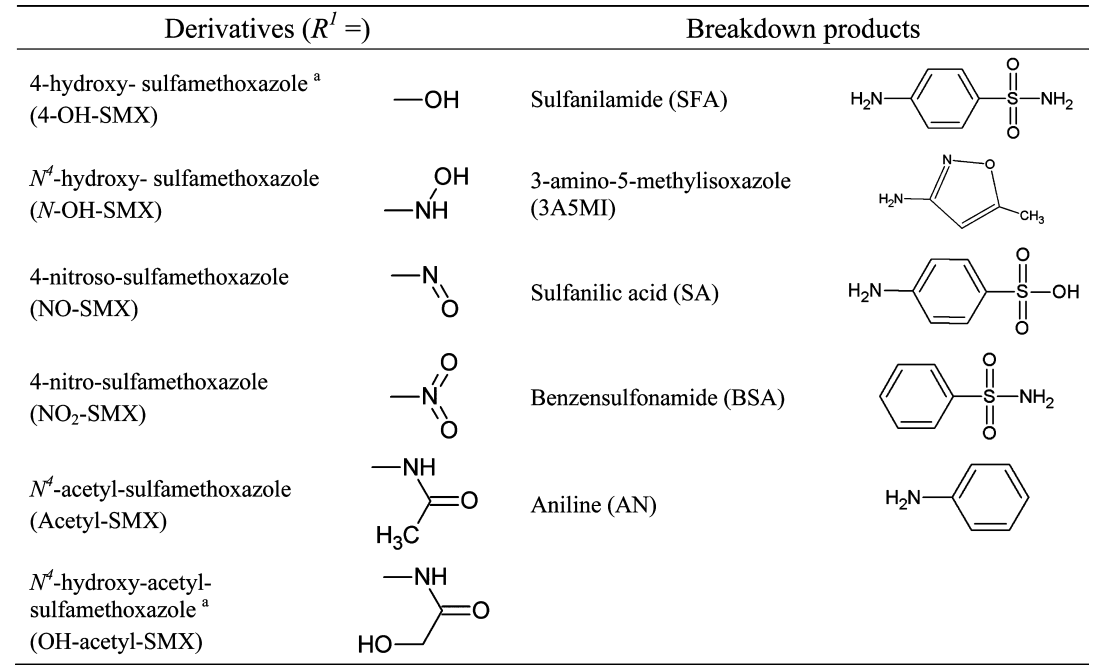

${ }^{a}$ Synthesized in this study. 
Table 2. $\mathrm{EC}_{50}$ Values for Luminescence Inhibition (LI) and Growth Inhibition (GI), Sulfamethoxazole Toxicity Equivalents $\left(\mathrm{TE}_{\mathrm{SMX}}\right)$, and Acute-to-Chronic Ratios (ACR) of Sulfamethoxazole and the Selected Transformation Products ${ }^{a}$

\begin{tabular}{|c|c|c|c|c|c|c|c|}
\hline & $\begin{array}{c}\mathrm{EC}_{50} \\
\mathrm{LI}_{30 \mathrm{~min}} \\
{\left[\mu \mathrm{mol} \mathrm{L}^{-1}\right]}\end{array}$ & $\begin{array}{c}\mathrm{EC}_{50} \\
\mathrm{LI}_{24 \mathrm{~h}} \\
{\left[\mu \mathrm{mol} \mathrm{L}^{-1}\right]}\end{array}$ & $\begin{array}{c}\mathrm{EC}_{50} \\
\mathrm{GI}_{24 \mathrm{~h}} \\
{\left[\mu \mathrm{mol} \mathrm{L}^{-1}\right]}\end{array}$ & $\begin{array}{c}\mathrm{TE}_{\mathrm{SMX}} \\
\mathrm{LI}_{30}\end{array}$ & $\begin{array}{c}\mathrm{TE}_{\mathrm{SMX}} \\
\mathrm{LI}_{24}\end{array}$ & $\begin{array}{c}\mathrm{TE}_{\mathrm{SMX}} \\
\mathrm{GI}_{24}\end{array}$ & ACR \\
\hline SMX & 551 & 7 & 599 & 1 & 1 & 1 & 79 \\
\hline \multicolumn{8}{|l|}{ Derivatives } \\
\hline $\mathrm{NO}_{2}$-SMX & 168 & 6 & 28 & 3.3 & 1.2 & 21.4 & 28 \\
\hline NO-SMX & 464 & 9 & 19822 & 1.2 & 0.8 & $<0.05$ & 52 \\
\hline 4-OH-SMX & 245 & 11 & 106 & 2.2 & 0.6 & 5.7 & 22 \\
\hline $\mathrm{N}$-OH-SMX & 1044 & 23 & 788 & 0.5 & 0.3 & 0.8 & 45 \\
\hline Acetyl-SMX & 3466 & 252 & 7567 & 0.2 & $<0.05$ & 0.1 & 14 \\
\hline OH-Acetyl-SMX & $>1333$ & $>1333$ & 5590 & $<0.4$ & $<0.05$ & 0.1 & \\
\hline \multicolumn{8}{|c|}{ Breakdown Products } \\
\hline SFA & 1006 & 900 & 23813 & 0.65 & 0.01 & 0.06 & 1 \\
\hline SA & 4057 & 2867 & 6854 & 0.16 & $<0.01$ & 0.21 & 1 \\
\hline BSA & 9534 & 2966 & 18687 & 0.07 & $<0.01$ & 0.08 & 3 \\
\hline AN & 9709 & 7780 & 24805 & 0.07 & $<0.01$ & 0.06 & 1 \\
\hline 3A5MI & $>5596$ & 2731 & $>5596$ & $<0.1$ & $<0.01$ & $<0.1$ & $>2$ \\
\hline
\end{tabular}

were purchased from Toronto Chemicals (Toronto, ON, Canada). NO-SMX was stored at $-20{ }^{\circ} \mathrm{C}$ due to its high reactivity, and the other compounds were kept at $+4{ }^{\circ} \mathrm{C}$, as recommended by suppliers. All chemicals exhibited purities $>95 \%$ except for NO-SMX, with $>90 \%$. Freeze-dried luminescent bacteria (Acute Reagent, 855-637-6426) were purchased from Modern Water (New Castle, DE, USA) and stored at $-20{ }^{\circ} \mathrm{C}$ until use. All chemicals used to synthesize TPs not commercially available for syntheses were purchased from SigmaAldrich (Germany).

2.2. Synthesis of OH-Acetyl-SMX and 4-OH-SMX. Synthesis of 4-OH-SMX was previously reported in the literature. ${ }^{22}$ However, a reproduction of the synthesis of 4-OH-SMX using the reported procedure did not yield satisfying results. Therefore, the desired product was synthesized in two steps via the previously unknown 4methoxy- $\mathrm{N}$-(5-methylisoxazol-3-yl)benzenesulfonamide and following cleavage of the methoxy group with boron tribromide. The detailed procedure, corresponding analytical nuclear magnetic resonance (NMR), and mass spectrometry data are given in Supporting Information S1. All synthesized compounds exhibit good purities (>95\%) according to ${ }^{1} \mathrm{H}$ and ${ }^{13} \mathrm{C}$ NMR. The purities are also supported by satisfying elemental analyses with the exception of 4$\mathrm{OH}-\mathrm{SMX}$, which contained traces of water that was difficult to remove.

2.2.1. 4-Hydroxy- $N$-(5-methylisoxazol-3-yl)benzenesulfonamide (4-OH-SMX). To a solution of 4-methoxy- $\mathrm{N}$-(5methylisoxazol-3-yl)benzenesulfonamide $(800 \mathrm{mg}, 2.98 \mathrm{mmol}, 1$ equiv) in dry dichloromethane $(15 \mathrm{~mL})$ at $-78{ }^{\circ} \mathrm{C}$ was added boron tribromide solution $(1 \mathrm{M}$ in dichloromethane, $8.95 \mathrm{~mL}, 8.95$ mmol, 3 equiv) dropwise under an inert atmosphere with stirring. After $30 \mathrm{~min}$, the reaction mixture was warmed to room temperature and stirred for a further $5 \mathrm{~h}$. It was then quenched and neutralized with a saturated $\mathrm{NaHCO}_{3}$ solution and extracted with ethyl acetate $(3 \times 30$ $\mathrm{mL}$ ). The organic phase was dried over anhydrous sodium sulfate, filtered, and concentrated under reduced pressure. Flash chromatography (silica gel, cyclohexane/ethyl acetate $1: 1 \mathrm{v} / \mathrm{v}$ ) of the crude product afforded $310 \mathrm{mg}$ (41\% yield) of the title compound as a yellow solid.

2.2.2. $N^{4}$-Hydroxyacetylsulfamethoxazole (OH-Acetyl-SMX). Sulfamethoxazole ( $700 \mathrm{mg} ; 2.76 \mathrm{mmol} ; 1$ equiv) was dissolved in dry tetrahydrofuran $(14 \mathrm{~mL})$ under an inert atmosphere. Triethylamine $(1.11 \mathrm{~mL} ; 8.02 \mathrm{mmol} ; 2.9$ equiv) and acetoxyacetyl chloride (1.02 g; $7.46 \mathrm{mmol} ; 2.7$ equiv) were added, and the reaction mixture was stirred at room temperature for $16 \mathrm{~h}$. It was then concentrated under reduced pressure. Subsequently, a $1.5 \mathrm{~N}$ aqueous sodium hydroxide solution $(20 \mathrm{~mL})$ and methanol $(20 \mathrm{~mL})$ were added to the resulting residue, and the mixture was again stirred at room temperature for 12 h. The reaction mixture was concentrated under reduced pressure, and the residue was then dissolved in water. The solution was neutralized with $1 \mathrm{~N}$ aqueous $\mathrm{HCl}$ and then extracted with ethyl acetate $(3 \times 30$ $\mathrm{mL}$ ). The organic layer was washed with saturated brine, dried over anhydrous sodium sulfate, and filtered. The filtrate was concentrated under reduced pressure, and the crude product was purified by column chromatography (silica gel, cyclohexane/ethyl acetate $1: 2$ to $0: 1$ ) to afford $670 \mathrm{mg}$ (78\% yield) of the title compound as yellowish crystals.

2.3. Bioassays. Short-term tests using LI end points, such as 15 or $30 \mathrm{~min}$, usually fall short of the delayed bacteriostatic effects of antibiotics and therefore can significantly underestimate the ecotoxicological effects. ${ }^{23,24}$ For this reason, long-term bioassays over $24 \mathrm{~h}$ were applied here by providing a growth medium with carbon and nutrient sources ${ }^{25}$ to sustain the bacteria and their luminescence light emission over this extended test duration. This test approach also allows bacterial growth to be tracked by optical density measurements in addition to luminescence light emission. ${ }^{26}$ A detailed composition of the growth medium used is given in Supporting Information S2. Apart from this, experiments were carried out according to the standard procedure ISO $11348-3^{27}$ for Vibrio fischeri and repeated in independent experiments for each compound. The initial $\mathrm{pH}$ was 6.8, and no further adjustments were made. Luminescence and optical density (OD) were measured after $30 \mathrm{~min}$ (end point most commonly applied) and $24 \mathrm{~h}$, respectively. Relative luminescence light emission was measured by a Microtox M500 Analyzer (Modern Water, New Castle, DE, USA; formerly SDI). Relative absorbance was measured by a $\mathrm{HACH}$ photometer at $\lambda=600 \mathrm{~nm}$. Pipette tips and glassware used for stock solutions and substrate were autoclaved for $20 \mathrm{~min}$ at $121{ }^{\circ} \mathrm{C}$ before usage to avoid microbial contamination. A detailed description of the procedure, the composition of the substrates, and test validation experiments regarding the interaction of SMX TPs with the growth substrate, possible light absorption, substrate competition, and comparison to ISO 11348-3 can be found in Supporting Information S2.

The Hill equation was used to fit the monotonic dose-response relationships obtained from the bioassays by minimizing $\chi^{2}$ between modeled and measured data using OriginPro 8.5 software (OriginLab Corporation, USA)

$$
I=I_{0}+\left(I_{\max }-I_{0}\right) \frac{c^{n}}{\mathrm{EC}_{50}^{n}+c^{n}}
$$

where $I=$ inhibition, $I_{0}=$ the minimum inhibition (set to $\left.I_{0}=0 \%\right), I_{\max }$ $=$ maximum inhibition (set to $I_{0}=100 \%$ ), $c=$ the toxicant concentration in micromoles per liter, $\mathrm{EC}_{50}=$ effective concentration at $I=50 \%$, and $n=$ Hill coefficient. 

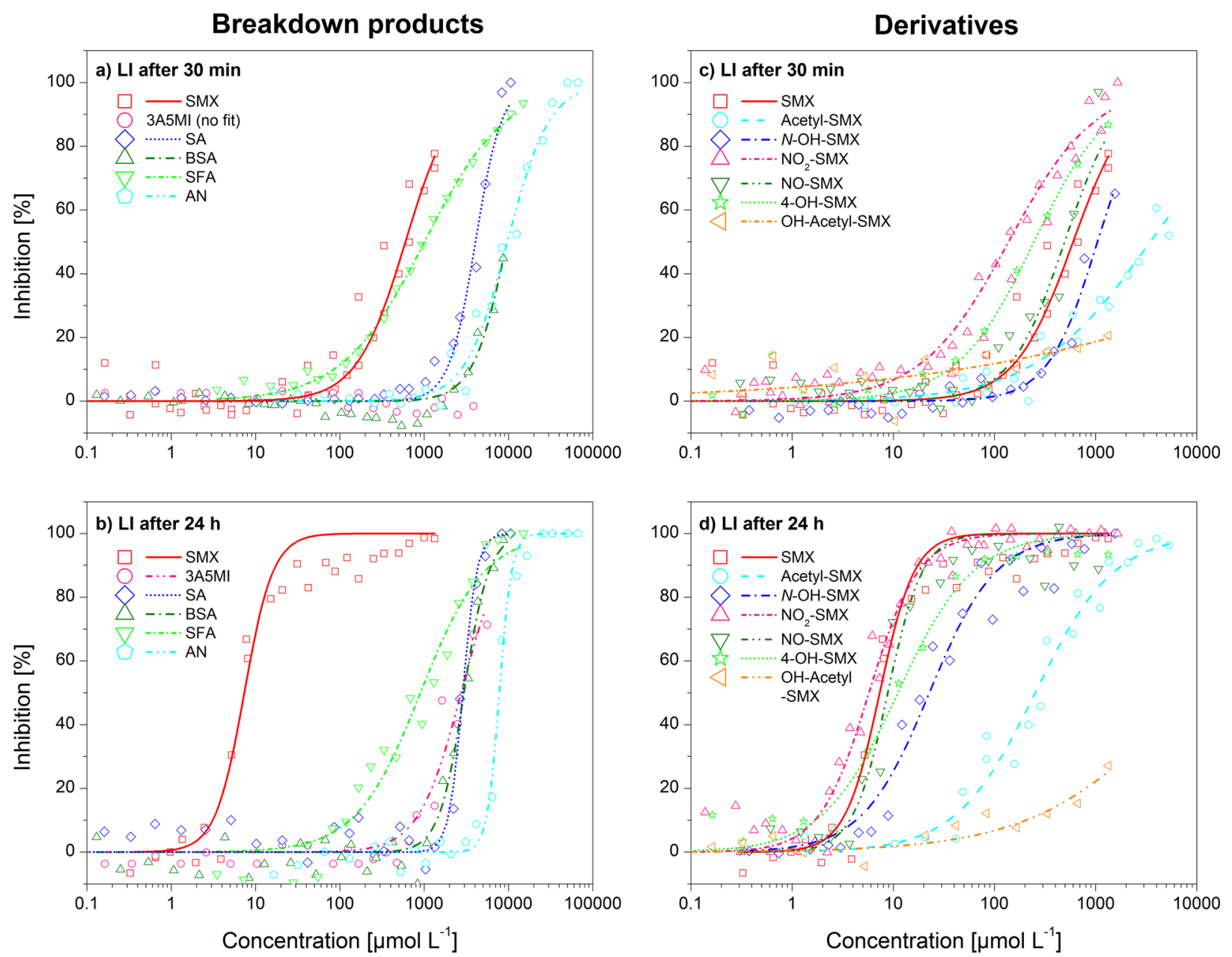

Figure 2. $(\mathrm{a}-\mathrm{d})$ Dose-response relationships of SMX TPs and V. fischeri (left panels, breakdown produts; right panels, derivatives; top panels, $\mathrm{LI}_{30 \text { min }}$; bottom panels, $\left.\mathrm{LI}_{24 \mathrm{~h}}\right)$. SMX is shown in all four graphs for comparison; data were fitted with the Hill equation; $n=15-39$. All $r^{2}>0.91$ except for OH-Ace-SMX $(0.24,0.62)$, indicating that the proportion of variation accounted for by the Hill model compared to the null model (mean of the $y$ values) is smaller than that for the other compounds; please note the different $x$-axis scales for breakdown products and derivatives.

2.4. Determination of $\mathrm{p} K_{\mathrm{a} 2}$. Acidic dissociation constants for the $N^{1}$ amino group $\left(\mathrm{p} K_{\mathrm{a} 2}\right)$ of the two synthesized metabolites, 4-OHSMX and OH-acetyl-SMX, as well as of $\mathrm{N}$-OH-SMX (not available in literature) were determined by measuring absorbance in titration experiments over a $\mathrm{pH}$ range from 2 to 10 using $\mathrm{HCl}$ and $\mathrm{NaOH}(c=$ $\left.0.05 \mathrm{~mol} \mathrm{~L}^{-1}\right)$ in tap water. Controls were run to check for possible interference with $\mathrm{HCO}_{3}{ }^{-} / \mathrm{CO}_{3}{ }^{2-}$. An automatic titrator (SI Analytics) was used to adjust the $\mathrm{pH}$, and absorbance measurements were carried out using a photometer (Cary 50, Varian) at $\lambda=235 \mathrm{~nm}$ for 4-OHSMX and OH-acetyl-SMX and at $\lambda=245 \mathrm{~nm}$ for $N$-OH-SMX. Concentrations of the TPs were $c=3 \mu \mathrm{mol} \mathrm{L}^{-1}$, and the temperature was $\vartheta=20^{\circ} \mathrm{C}$. Wavelengths were selected on the basis of the UV/vis spectra $(200-400 \mathrm{~nm})$ at $\mathrm{pH} 3$ and 11. Experimental data were fitted with the sigmoidal Boltzmann equation according to Bonvin et al. ${ }^{8}$ For the $\mathrm{pH}$ range typically prevailing in aquatic environments, only the $\mathrm{p} K_{\mathrm{a} 2}$ is relevant. Experimental $\mathrm{p} K_{\mathrm{a} 2}$ values for SMX and the other TPs were taken from the literature, and theoretical $\mathrm{p} K_{\mathrm{a} 2}$ values for all compounds were also calculated for comparison using Marvin Sketch (ChemAxon, Hungary) (Table S1, Supporting Information).

\section{RESULTS}

3.1. Growth and Luminescence Test Validation. Compared to the ISO test conditions using only a salty test medium, the approach applied here used additional carbon and nutrient sources to ensure luminescence light emission over a longer period and to allow $V$. fischeri to grow. Both are inherently linked, since around $10 \%$ of the metabolic energy is converted into luminescence light production ${ }^{28}$ and in this way is related to cellular respiration. A series of validation experiments with SMX and SFA, as representatives for ionized and neutral compounds, showed that the growth medium does not interfere with the test results. Testing of SMX with the growth medium provided a dose-response relationship matching the one obtained using ISO 11348-3 (Figure S3). The detailed data and further results of the validation experiments are given in Supporting Information S3.

3.2. Dose-Response Relationships of SMX TPs. 3.2.1. Luminescence Inhibition (LI). All breakdown products were more than 2 to 3 orders of magnitude less active than SMX, resulting in $\mathrm{EC}_{50}$ values between 900 and $9709 \mu \mathrm{mol} \mathrm{L}{ }^{-1}$, which correspond to the upper milligram per liter mass concentration range (Table 2). 3A5MI, which was reported to be a dead-end metabolite of $\mathrm{SMX}^{13}$ and thus may accumulate in the environment, showed $\mathrm{LI}_{24 \mathrm{~h}}$ at $\mathrm{EC}_{50}=2.7$ mmol L ${ }^{-1}\left(264.9 \mathrm{mg} \mathrm{L}^{-1}\right)$, whereas no effect was observable after $30 \mathrm{~min}$. The ratio of both LI end points, termed here as the acute-to-chronic ratio (ACR), is between 1 and 3 for the breakdown products, i.e., their $\mathrm{EC}_{50}$ at both test durations changed only marginally (Figure 2a,b). 
The picture is substantially different for SMX and its derivatives (Table 2). As can be seen in Figure 2c,d, results for $\mathrm{LI}$ after $30 \mathrm{~min}$ and $24 \mathrm{~h}$ differed considerably for these two end points for both SMX and its derivative TPs. The ACRs show that the $\mathrm{EC}_{50}$ for these compounds were 14-79 times lower after $24 \mathrm{~h}$ than after $30 \mathrm{~min}$. This indicates their bacteriostatic mechanism of action, whose effect becomes apparent only after many microbial reproduction cycles. These results are in agreement with the findings previously reported for other parent antibiotics by Backhaus et al. ${ }^{29}$ No ACR value could be derived for $\mathrm{OH}$-acetyl-SMX since its $\mathrm{LI} \mathrm{EC}_{50}$ values were not reached for both exposure times.

The $\mathrm{LI}_{24 \mathrm{~h}} \mathrm{EC}_{50}$ of SMX was $7 \mu \mathrm{mol} \mathrm{L} \mathrm{L}^{-1}\left(1.7 \mathrm{mg} \mathrm{L}^{-1}\right)$. Expressing the toxicity of the derivatives relative to SMX as toxicity equivalents $\left(\mathrm{TE}_{\mathrm{SMX}}\right)$, it can be seen that $\mathrm{NO}_{2}-\mathrm{SMX}$ and NO-SMX are similarly toxic as the parent compound, followed by $4-\mathrm{OH}-$ and $\mathrm{N}-\mathrm{OH}-\mathrm{SMX}$, whereas the two acetylated derivatives seem to exhibit very low antibacterial activity after 24 h. After 30 min, NO-SMX, 4-OH-SMX, and $\mathrm{NO}_{2}$-SMX even acted 1.2 to 3.3 times more toxic upon luminescence emission, which can, however, be attributed to an immediate interference with cellular respiration but not to a bacteriostatic effect due to the short exposure time.

3.2.2. Growth Inhibition (GI). Growth was generally less affected by the tested compounds than LI, as indicated by the clearly higher $\mathrm{GI} \mathrm{EC}_{50}$ values. GI could be measured only after $24 \mathrm{~h}$ since no significant growth occurs within $30 \mathrm{~min}$. All breakdown products show considerably lower toxicity by 1 to 2 orders of magnitude as compared to that of SMX (Table 2 and Figures S5 and S6 in the Supporting Information), indicating again the loss of the bacteriostatic mechanism of action. 3A5MI did not show any GI effects for the tested concentration range of up to $5.6 \mathrm{mmol} \mathrm{L}^{-1}\left(549 \mathrm{mg} \mathrm{L}^{-1}\right)$.

Furthermore, the two acetylated TPs are rather inactive toward GI, with $\mathrm{EC}_{50}$ values of 7.6 and $5.6 \mathrm{mmol} \mathrm{L}^{-1}$, respectively, which corresponds to less than $10 \%$ of the residual activity of SMX. On the contrary, the 4-OH and $\mathrm{NO}_{2}$ derivatives are 5.7 and 21.4 times more toxic relative to SMX, respectively. Also, $\mathrm{N}$-OH-SMX exhibited a GI effect of around $80 \%$ that of SMX. For this end point, the order of toxicity relative to SMX among the derivatives has changed to (listed by their $\mathrm{R}^{1}$ ) $\mathrm{NO}_{2^{-}}>$4-OH- $>\mathrm{NH}_{2^{-}}$(parent) $>\mathrm{N}-\mathrm{OH}->$ $\mathrm{N}$-acetyl-, $\mathrm{N}$-OH-acetyl- > NO-. NO-SMX exhibited a surprisingly low GI when compared to its $\mathrm{LI}_{30 \min }$ and $\mathrm{LI}_{24 \mathrm{~h}}$ values. It is known that NO-SMX is reactive and very unstable in solutions and therefore the high $\mathrm{GI} \mathrm{EC}_{50}$ values are likely to be due to decomposition or further transformation of the compound..$^{30}$ The different order of toxicity observed with GI implies that LI cannot be used as a proxy in order to predict the GI with sufficient certainty even when ignoring the results of NO-SMX. Moreover, no significant correlation could be found between ACR and GI. Although luminescence is clearly adversely affected by the TPs through GI, it is not the sole mechanism, as shown by the $\mathrm{EC}_{50}$ after $30 \mathrm{~min}$, during which no growth or GI took place.

3.3. Mixture Toxicity. Given the results of the experiments with the para-modified TPs compounds and their presumably similar mechanism of action, it was expected that the effect of a mixture of SMX derivatives can be described by the concept of concentration addition (CA), ${ }^{31}$ where the effect of a mixture is the sum of its effects at given individual compound concentrations. The CA approach is theoretically expressed as

$$
\mathrm{EC}_{x, \text { mix }}=\left(\sum_{i=1}^{n} \frac{p_{i}}{\mathrm{EC}_{x_{i}}}\right)^{-1}
$$

where $\mathrm{EC}_{x, \text { mix }}$ is the effective concentration where $x \%$ is affected by the mixture, $n$ is the number of compounds in the mixture, $p_{i}=$ proportion of each single compound concentration to the total concentration, and $\mathrm{EC}_{x_{i}}=$ the effective concentration of the single compound at a given effected fraction of $x \%$.

It was assumed that the tested compounds neither interacted with each other on a molecular level nor with regard to their toxicokinetics. As can be seen from Figure 3, the CA concept

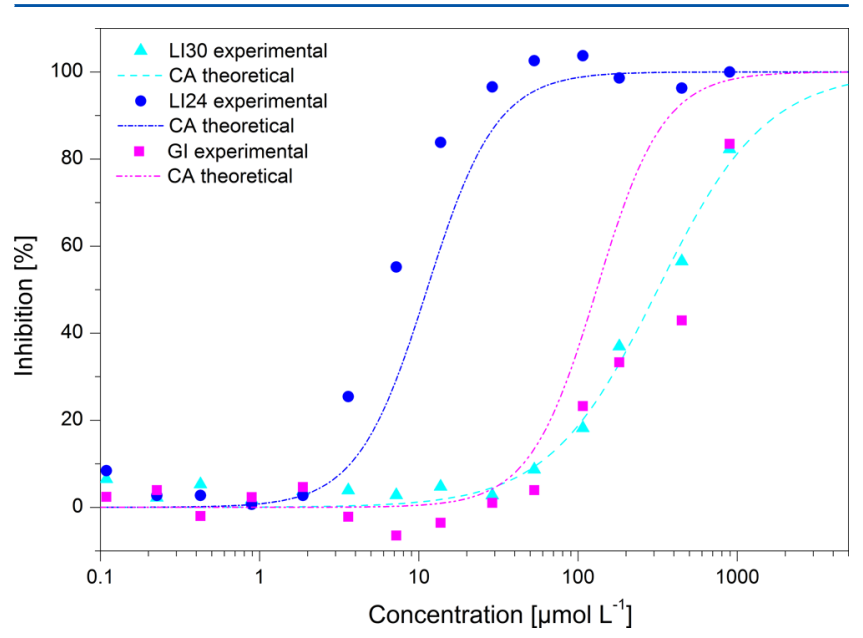

Figure 3. Experimental mixture toxicity of $\mathrm{SMX}, \mathrm{NO}_{2}-\mathrm{SMX}, \mathrm{NO}-$ SMX, $N$-OH-SMX, and $N$-acetyl-SMX as well as their theoretical mixture toxicity according to the concentration addition concept (CA, eq 2); starting concentration $c=0.8 \mathrm{mmol} \mathrm{L}^{-1}$ per compound in mixture.

was well-suited to describe the effect of an equimolar mixture of five compounds (SMX, $\mathrm{NO}_{2}$-SMX, NO-SMX, N-OH-SMX, and $N$-acetyl-SMX) for both LI exposure times and to a lesser extent for GI, where the model overestimates the experimental values.

We first hypothesized that the differences observed might be due to transformation of unstable NO-SMX, which might result in the formation of subproducts having an impact on luminescence but not on growth. However, a recalculation of the CA model without NO-SMX did not fully explain the differences between the observed and modeled GI data. The discrepancies are then most likely associated with experimental accuracy, leading to higher experimental uncertainty for GI as compared to that for LI.

Nonetheless, these results indicate that the derivative transformation products of SMX can contribute to the total antibacterial effect and therefore need to be considered in addition to the parent compound. Taking this one step further, it is relevant to assume that this applies to any sulfonamide present in a water body due to their common molecular structure and mechanism of action (cf. section 4, Discussion).

\section{DISCUSSION}

\subsection{Relationship between Antibacterial Activity and} para Transformations. Results showed that the toxicity of the breakdown products SFA and BSA, which both feature the 
benzenesulfonamide moiety, is considerably lower compared to SMX. Although the sulfanilamide building block is the toxicophore isosteric to $\mathrm{pABA}$, the low toxicity of the latter two compounds can be attributed to their presence as a neutral species at $\mathrm{pH} 6.8$ with $\mathrm{p} K_{\mathrm{a}}$ 's of 10.58 and 10.10 , respectively. In addition, BSA lacks the primary amino group at the para position, which is supposed to be paramount to interference with folic acid synthesis by binding to 6-methylpterin. Further details on the interaction of sulfonamides with the microbial folic acid pathway can be found elsewhere. ${ }^{32}$ In contrast, $p A B A$ exhibits a $\mathrm{pK}_{\mathrm{a} 2}$ of around 4.7 and thus is present in its negatively charged species to $99 \%$ at $\mathrm{pH}$ 6.8. This agrees with the findings by Hansch, ${ }^{33}$ who concluded from quantitative structure-activity relationship (QSAR) studies with Escherichia coli that the ionized amide group of the sulfonamide moiety promotes the biological effects.

All breakdown products, including the latter two, however, still provoke GI and LI at high doses, $>0.9 \mathrm{mmol} \mathrm{L}^{-1}$. This and the fact that there is no delayed effect on LI, as indicated by the ACRs, suggest another mechanism of action, most likely of a nonspecific narcosis type. In this regard, the $\mathrm{LI}_{30 \mathrm{~min}}$ and $\mathrm{LI}_{24 \mathrm{~h}}$ of the four breakdown products originating from the 4-aminobenzenesulfonamide moiety (SA, SFA, AN, and BSA) also showed a positive correlation with their log $K_{\mathrm{ow}}$ (Table S1, Supporting Information).

In view of the results of single substance and mixture toxicity experiments, the SMX derivatives are supposed to act via the same mechanism of action as SMX. Together, they form a homologous series with different substituents in the para position. QSAR have been established for parent sulfonamide homologues that unraveled their mechanisms of action. ${ }^{34}$ However, in these studies, only the $\mathrm{R}^{2}$ moiety was modified, whereas the $p$-amino group remained because, up to now, this group has been considered relevant for their mechanism of action. For instance, Nouws et al. ${ }^{35}$ concluded from the antibiotic inactivity of $N^{4}$-acetyl-sulfonamides on $E$. coli that a free $p$-aminophenyl group is required. To our knowledge, the results of the present study showed for the first time that SMX transformed at the para position can still exhibit and even increase its ecotoxicity. To explain this phenomenon, two mechanisms of action come into consideration: (i) competitive inhibition of the active site of the key enzyme dihydropteroate synthase (DHPS) for folic acid synthesis and/or (ii) incorporation of the TP in lieu of $p A B A$, which leads to inactive products.

By analogy to the parent sulfonamides, we first hypothesized different degrees of ionization of the TP and changes in the electron distribution (expressed as their $\mathrm{p} K_{\mathrm{a} 2}$ values) at the given $\mathrm{pH}$ to be responsible for the measured effects, which would indicate competitive protein binding. In order to test this assumption, the $\mathrm{p} K_{\mathrm{a} 2}$ values of the two synthesized metabolites as well as $\mathrm{N}-\mathrm{OH}-\mathrm{SMX}$ needed to be experimentally determined since no data were available in the literature (Table 3 ).

Plotting the experimental $\mathrm{p} K_{\mathrm{a} 2}$ values versus the $\mathrm{GI} \mathrm{EC}_{50}$ end points showed increasing toxicity with increasing $\mathrm{p} K_{\mathrm{a} 2}$ values and thus a decreasing degree of ionization for NO-, $\mathrm{N}$-acetyl-, $\mathrm{N}-\mathrm{OH}$-acetyl-SMX, and SMX itself (Figure 4). These data match well the data reported in literature for parent sulfonamides, which indicated an optimum at around $\mathrm{p} K_{\mathrm{a} 2}=$ 6.5 , before and after which the activity decreases. ${ }^{34,36}$ The data in this study present only the lower branch of the optimum, since all SMX-TPs exhibit $\mathrm{pK}_{\mathrm{a} 2}<5.9$. However, three derivatives are significantly more growth inhibiting than
Table 3. Experimental $\mathrm{p} K_{\mathrm{a} 2}$ Values of 4-Hydroxysulfamethoxazole, $\mathrm{N}^{4}$-Hydroxy-acetyl-sulfamethoxazole, and $N^{4}$-Hydroxy-sulfamethoxazole ${ }^{a}$

\begin{tabular}{lcccc}
\multicolumn{1}{c}{ compound } & $\mathrm{pK}_{\mathrm{a} 2}$ & confidence bands (95\%) & $n$ & $r^{2}$ \\
4-OH-SMX & 4.89 & \pm 0.09 & 24 & 0.97 \\
OH-Acetyl-SMX & 5.43 & \pm 0.05 & 24 & 0.99 \\
N-OH-SMX & 4.51 & \pm 0.04 & 17 & 0.99
\end{tabular}

${ }^{a}$ Data fitted with Boltzmann equation; see Figure S7, Supporting Information.

expected, namely, $\mathrm{NO}_{2^{-}}, \mathrm{N}-\mathrm{OH}$, and 4-OH-SMX. It should be noted that the differences among these three compounds are observable only when using the experimental $\mathrm{p} K_{\mathrm{a} 2}$ values, which were up to $2 \mathrm{p} K_{\mathrm{a} 2}$ units lower than the theoretical ones. The discrepancies between the modeled and measured values have been discussed in the literature and are therefore not repeated at this point.

The substituents of these three derivatives exert a negative inductive effect, which facilitates the release of the proton of the amide group and thus may result in a stronger affinity to bind to DHPS. However, these are also stronger nucleophiles than the common $p$-amino group of the sulfonamides, which may lead to an increased amount of faulty products by the replacement of $p A B A$ by covalent binding. Yun et al. ${ }^{37}$ showed that $p$-hydroxybenzoic acid ( $p \mathrm{HBA})$, which is structurally similar to 4-OH-SMX, bound to the active site of the responsible enzyme. Nevertheless, the exact mechanism cannot be elucidated by the data from this study.

4.2. General Relevance for para-Modified Sulfonamide TPs. The ecotoxicological screening using $V$. fischeri presented in this study can certainly be only a small contribution to a holistic environmental risk assessment. It requires testing for other bacterial species and higher trophic levels as well as data on the occurrence and persistence in wastewater and surface water of these compounds, which were out of scope of this study. However, on the basis of this screening and considering that SMX is a representative example of sulfonamide antibiotics, the question inevitably arises about the importance of considering para TPs formed from other homologous sulfonamides.

All TP SMX derivatives considered in this study underwent transformation at the para binding site, a site that is present in almost all sulfonamide antibiotics with only very few exceptions. Accepting the hypothesis that the ionization of the secondary amide largely promotes their biological effects, transformation reactions of sulfonamide homologues with electron-withdrawing moieties, such as nitro or hydroxyl groups, are generally suggested to be ecotoxicologically relevant. The para TPs still might act as a pABA substitute, which leads to inactive products, i.e., that these TPs exhibit some relevant ecotoxicity of the parent compound toward $V$. fischeri and most likely also toward other bacterial species. The acetylated TPs showed very low toxicity relative to SMX $(<10 \%)$ for both GI and LI. The environmental relevance of these derivatives is, however, difficult to assess considering that these TPs can potentially be transformed back to the parent compound. $^{8}$

As shown in this study, the $\mathrm{R}^{2}$ moieties alone released from sulfonamide bonding cleavage can be assumed to be of minor ecotoxicological relevance with regard to their antibacterial effects. Consequently, focus has to be put on the derivatives presumably bearing antibiotic potential. However, it cannot 


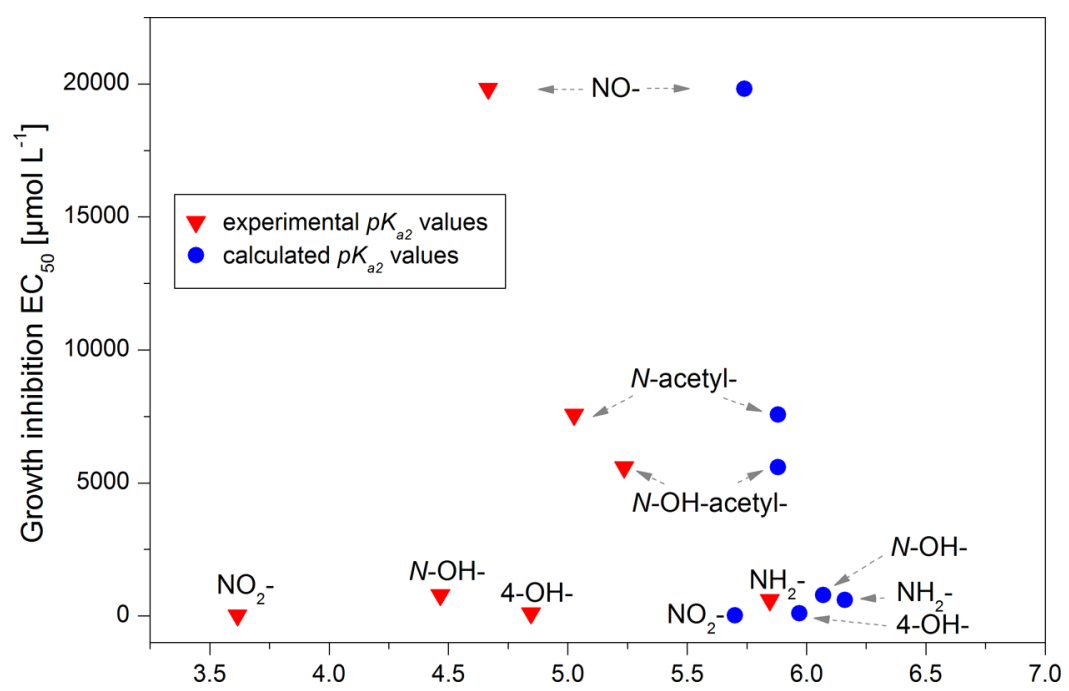

Figure 4. Plotting of the acidic constants $\mathrm{p} K_{\mathrm{a} 2}$ (theoretical and experimental $\mathrm{p} K_{\mathrm{a} 2}$ values) versus $\mathrm{GI} \mathrm{EC}_{50}$; experimental $\mathrm{p} K_{\mathrm{a} 2}$ values taken from literature as well as theoretical values are listed in Table S1.

ultimately be excluded that the $\mathrm{R}^{2}$ moieties exhibit toxicological activity upon species other than bacteria and/or through other mechanisms of action.

The concept of concentration addition suggests that the effects of all compounds with this mechanism of action in an aquatic system sum. As a consequence, a profound ecological risk assessment principally needs to consider all sulfonamides and derivative TPs present in an aquatic system. Realistically, their cumulative amount acts as the key factor for the possible evolution or preservation of microbial antibiotic resistance. Coping with this issue, one option consists of a combined approach by quantifying the total impact via sum parameters, such as biomarkers ${ }^{32}$ or sulfonamide ELISA as well as prioritization and determination of single relevant TPs.

Bearing in mind that extrapolation from in vitro to in vivo has its restrictions and considering that it seems unlikely that $\mathrm{EC}_{50}$ values of GI might be observed for individual compounds or mixtures in the aquatic environment (lowest GI values were in the milligrams per liter range), the risk posed by these compounds seems limited. Moreover, knowing that $V$. fischeri is a marine species, testing of further species native to surface water habitats is advisable. However, recent research has given insight into sulfonamide-resistance mechanisms, suggesting that the latter is associated with the $\mathrm{R}^{2}$ moieties in the sulfa drug, which have no equivalent in $p \mathrm{ABA}$ and are located outside the DHPS substrate envelope where mutations may impede sulfa drug binding. ${ }^{37}$ In this regard, the derivative TPs should be of equal interest as the parent compound as they may promote antibiotic resistance in the same way. To our knowledge, it was observed for the first time that sulfonamides act via their bacteriostatic mechanism of action even though they did not feature the free primary amino group. As for the breakdown products, SA, SFA, AN, and BSA, which are common to all sulfonamides as well as the $\mathrm{R}^{2}$ moiety $3 \mathrm{~A} 5 \mathrm{MI}$, they all exhibited low GI and LI values.

These results provided insight into the potential environmental impact of para-substituted TPs of SMX, but still relatively little is known about their occurrence, persistence, and origin. Further monitoring is needed to ensure that the risk of sulfa drugs and their TPs is not underestimated by focusing only on the parent compounds.

\section{ASSOCIATED CONTENT}

\section{S Supporting Information}

Experimental validation; NMR, HRMS, and IR data; $\mathrm{p} K_{\mathrm{a} 2}$ value determination. This material is available free of charge via the Internet at http://pubs.acs.org.

\section{AUTHOR INFORMATION}

\section{Corresponding Author}

*E-mail: marius.majewsky@partner.kit.edu; Tel.: +49 721 60842788.

\section{Funding}

We thank the Karlsruhe House of Young Scientists (KHYS) of the Karlsruhe Institute of Technology (KIT), the German Technical and Scientific Association for Gas and Water (DVGW), and the Natural Sciences and Engineering Research Council of Canada (NSERC) for their financial support.

\section{Notes}

The authors declare no competing financial interest.

\section{ABBREVIATIONS}

ACR, acute-to-chronic-ratio; CA, concentration addition; DHPS, dihydropteroate synthase; EC, effective concentration; ELISA, enzyme linked immunosorbent assay; GI, growth inhibition; HRMS, high-resolution mass spectrometry; LI, luminescence inhibition; NMR, nuclear magnetic resonance; OD, optical density; pABA, $p$-aminobenzoic acid; PCPP, pharmaceuticals and personal care products; $p \mathrm{HBA}, p$ hydroxybenzoic acid; QSAR, quantitative structure-activity relationships; SMX, sulfamethoxazole; 4-OH-SMX, 4-hydroxysulfamethoxazole; $\mathrm{N}$-OH-SMX, $\mathrm{N}^{4}$-hydroxy-sulfamethoxazole; NO-SMX, 4-nitroso-sulfamethoxazole; $\mathrm{NO}_{2}$-SMX, 4-nitrosulfamethoxazole; acetyl-SMX, $N^{4}$-acetyl-sulfamethoxazole; $\mathrm{OH}$-acetyl-SMX, $\mathrm{N}^{4}$-hydroxy-acetyl-sulfamethoxazole; SFA, sulfanilamide; 3A5MI, 3-amino-5-methylisoxazole; SA, sulfanilic acid; BSA, benzensulfonamide; $\mathrm{AN}$, aniline; $\mathrm{TE}_{\mathrm{SMX}}$, sulfamethoxazole toxicity equivalents; TP, transformation product

\section{REFERENCES}

(1) Heberer, T. (2002) Occurrence, fate, and removal of pharmaceutical residues in the aquatic environment: a review of recent research data. Toxicol. Lett. 131, 5-17. 
(2) Rosi-Marshall, E. J., Kincaid, D. W., Bechtold, H. A., Royer, T. V., Rojas, M., and Kelly, J. J. (2013) Pharmaceuticals suppress algal growth and microbial respiration and alter bacterial communities in stream biofilms. Ecol. Appl. 23, 583-593.

(3) De Bel, E., Dewulf, J., Witte, B. D., Van Langenhove, H., and Janssen, C. (2009) Influence of $\mathrm{pH}$ on the sonolysis of ciprofloxacin: biodegradability, ecotoxicity and antibiotic activity of its degradation products. Chemosphere 77, 291-295.

(4) Escher, B. I., and Fenner, K. (2011) Recent advances in environmental risk assessment of transformation products. Environ. Sci. Technol. 45, 3835-3847.

(5) Wammer, K. H., Slattery, M. T., Stemig, A. M., and Ditty, J. L. (2011) Tetracycline photolysis in natural waters: loss of antibacterial activity. Chemosphere 85, 1505-1510.

(6) Tamtam, F., Mercier, F., Le Bot, B., Eurin, J., Tuc Dinh, Q., Clement, M., and Chevreuil, M. (2008) Occurrence and fate of antibiotics in the Seine River in various hydrological conditions. Sci. Total Environ. 393, 84-95.

(7) Watkinson, A. J., Murby, E. J., Kolpin, D. W., and Costanzo, S. D. (2009) The occurrence of antibiotics in an urban watershed: from wastewater to drinking water. Sci. Total Environ. 407, 2711-2723.

(8) Bonvin, F., Omlin, J., Rutler, R., Schweizer, W. B., Alaimo, P. J., Strathmann, T. J., McNeill, K., and Kohn, T. (2012) Direct photolysis of human metabolites of the antibiotic sulfamethoxazole: evidence for abiotic back-transformation. Environ. Sci. Technol. 47, 6746-6755.

(9) García-Galán, M. J., Silvia Díaz-Cruz, M., and Barceló, D. (2008) Identification and determination of metabolites and degradation products of sulfonamide antibiotics. TrAC, Trends Anal. Chem. 27, $1008-1022$

(10) Larcher, S., and Yargeau, V. (2011) Biodegradation of sulfamethoxazole by individual and mixed bacteria. Appl. Microbiol. Biotechnol. 91, 211-218.

(11) Larcher, S., and Yargeau, V. (2013) The effect of ozone on the biodegradation of $17 \alpha$-ethinylestradiol and sulfamethoxazole by mixed bacterial cultures. Appl. Microbiol. Biotechnol. 97, 2201-2210.

(12) Nödler, K., Licha, T., Barbieri, M., and Pérez, S. (2012) Evidence for the microbially mediated abiotic formation of reversible and non-reversible sulfamethoxazole transformation products during denitrification. Water Res. 46, 2131-2139.

(13) Müller, E., Schüssler, W., Horn, H., and Lemmer, H. (2013) Aerobic biodegradation of the sulfonamide antibiotic sulfamethoxazole by activated sludge applied as co-substrate and sole carbon and nitrogen source. Chemosphere 92, 969-978.

(14) Boreen, A. L., Arnold, W. A., and McNeill, K. (2004) Photochemical fate of sulfa drugs in the aquatic environment: sulfa drugs containing five-membered heterocyclic groups. Environ. Sci. Technol. 38, 3933-3940.

(15) Periša, M., Babić, S., Škorić, I., Frömel, T., and Knepper, T. (2013) Photodegradation of sulfonamides and their $N^{4}$-acetylated metabolites in water by simulated sunlight irradiation: kinetics and identification of photoproducts. Environ. Sci. Pollut. Res. 20, 89348946.

(16) Dodd, M. C., Kohler, H.-P. E., and von Gunten, U. (2009) Oxidation of antibacterial compounds by ozone and hydroxyl radical: elimination of biological activity during aqueous ozonation processes. Environ. Sci. Technol. 43, 2498-2504.

(17) Trovo, A. G., Nogueira, R. F., Aguera, A., Sirtori, C., and Fernandez-Alba, A. R. (2009) Photodegradation of sulfamethoxazole in various aqueous media: persistence, toxicity and photoproducts assessment. Chemosphere 77, 1292-1298.

(18) Ashton, D., Hilton, M., and Thomas, K. V. (2004) Investigating the environmental transport of human pharmaceuticals to streams in the United Kingdom. Sci. Total Environ. 333, 167-184.

(19) Boxall, A. B. A., Sinclair, C. J., Fenner, K., Kolpin, D., and Maund, S. J. (2004) When synthetic chemicals degrade in the environment. Environ. Sci. Technol. 38, 368A-375A.

(20) Schwartz, T., Kohnen, W., Jansen, B., and Obst, U. (2003) Detection of antibiotic-resistant bacteria and their resistance genes in wastewater, surface water, and drinking water biofilms. FEMS Microbiol. Ecol. 43, 325-335.

(21) Gauthier, H., Yargeau, V., and Cooper, D. G. (2010) Biodegradation of pharmaceuticals by Rhodococcus rhodochrous and Aspergillus niger by co-metabolism. Sci. Total Environ. 408, 1701-1706.

(22) Vidyasagar, A., Daye, A. M., Mehta, M. H., and Agrawal, Y. K. (1991) Synthesis and antifungal activity of some N-substitutedbenzenesulphonamides pendant with 2-thiol-1,3,4-oxadiazoles, 3mercapto-4-phenyl-1,2,4(H)-triazoles. J. Indian Chem. Soc. 68, 576578.

(23) Backhaus, T., and Grimme, L. H. (1999) The toxicity of antibiotic agents to the luminescent bacterium Vibrio fischeri. Chemosphere 38, 3291-3301.

(24) Kümmerer, K. (2009) Antibiotics in the aquatic environmenta review-part I. Chemosphere 75, 417-434.

(25) Fröhner, K., Meyer, W., and Grimme, L. H. (2002) Timedependent toxicity in the long-term inhibition assay with Vibrio fischeri. Chemosphere 46, 987-997.

(26) Menz, J., Schneider, M., and Kümmerer, K. (2013) Toxicity testing with luminescent bacteria-characterization of an automated method for the combined assessment of acute and chronic effects. Chemosphere 93, 990-996.

(27) ISO 11348-3:2007 (2007) Water quality-Determination of the inhibitory effect of water samples on the light emission of Vibrio fischeri (luminescent bacteria test)—Part 3: Method using freeze-dried bacteria, International Organization for Standardization, Geneva, Switzerland.

(28) Klopman, G., and Stuart, S. E. (2003) Multiple computerautomated structure evaluation study of aquatic toxicity. III. Vibrio fischeri. Environ. Toxicol. Chem. 22, 466-472.

(29) Backhaus, T., Froehner, K., Altenburger, R., and Grimme, L. H. (1997) Toxicity testing with Vibrio fischeri: a comparison between the long term $(24 \mathrm{~h})$ and the short term $(30 \mathrm{~min})$ bioassay. Chemosphere $35,2925-2938$.

(30) Naisbitt, D. J., O’Neill, P. M., Pirmohamed, M., and Kevin Park, B. (1996) Synthesis and reactions of nitroso sulphamethoxazole with biological nucleophiles: implications for immune mediated toxicity. Bioorg. Med. Chem. Lett. 6, 1511-1516.

(31) Backhaus, T., and Faust, M. (2012) Predictive environmental risk assessment of chemical mixtures: a conceptual framework. Environ. Sci. Technol. 46, 2564-2573.

(32) Richter, M. K., Focks, A., Siegfried, B., Rentsch, D., Krauss, M., Schwarzenbach, R. P., and Hollender, J. (2013) Identification and dynamic modeling of biomarkers for bacterial uptake and effect of sulfonamide antimicrobials. Environ. Pollut. 172, 208-215.

(33) Hansch, C. (2003) Comparative QSAR of the sulfonamide function. Farmaco 58, 625-629.

(34) Seydel, J. K. (1981) Mode of action and quantitative structureactivity relationship of sulfonamides in biological systems of different complexity. Int. J. Quantum Chem. 20, 131-150.

(35) Nouws, J. F. M., Vree, T. B., and Hekster, Y. A. (1985) In vitro antimicrobial activity of hydroxy and $\mathrm{N}^{4}$-acetyl sulphonamide metabolites. Vet. Q. 7, 70-72.

(36) Bell, P. H., and Roblin, R. O., Jr. (1942) Studies in chemotherapy. VII. A theory of the relation of structure to activity of sulfonamide type compounds. J. Am. Chem. Soc. 64, 2905-2917.

(37) Yun, M. K., Wu, Y., Li, Z., Zhao, Y., Waddell, M. B., Ferreira, A. M., Lee, R. E., Bashford, D., and White, S. W. (2012) Catalysis and sulfa drug resistance in dihydropteroate synthase. Science 335, 11101114. 